\title{
QUILT: A Decode/Quantize-Interleave-Transmit Approach to Cooperative Relaying
}

\author{
Siddhartha Brahma*1, Melissa Duarte*1, Ayan Sengupta*1, I-Hsiang Wang ${ }^{1}$, Christina Fragouli ${ }^{1}$, Suhas Diggavi ${ }^{2}$ \\ ${ }^{1}$ EPFL, Switzerland, ${ }^{2}$ UCLA, USA \\ ${ }^{*}$ Co-First Authors (Alphabetical)
}

\begin{abstract}
Physical layer cooperation of a source with a relay can significantly boost the performance of a wireless connection. However, the best practical relaying scheme can vary depending on the relative strengths of the channels that connect the source, relay and destination. This paper proposes and evaluates QUILT, a system for physical-layer relaying that seamlessly adapts to the underlying network configuration to achieve competitive or better performance as compared to the best current approaches. QUILT combines on-demand, opportunistic use of Decode-Forward (DF) or Quantize-Map-Forward (QMF) followed by interleaving at the relay, with hybrid decoding at the destination that extracts information of received frames even if these are not decodable. We theoretically quantify how our design choices for QUILT affect the system's performance. We also deploy QUILT on the WarpLab software radio platform, and show through over the over-the-air experiments up to 5 times FER improvement over the next best cooperative protocol.
\end{abstract}

\section{INTRODUCTION}

Physical layer cooperation of a source with a relay can significantly boost the performance of a wireless system. This is supported from theoretical works that have proposed a number of relay cooperation schemes [1], verified by first experimental results [2] and recognized by the standards that are starting to include relaying mechanisms [3].

A natural question is, which scheme performs better when deployed in a practical system. In our recent work [2], we implemented the most promising state-of-the-art relaying schemes - Amplify-Forward (AF), Decode-Forward (DF) and Quantize-Map-Forward (QMF) on top of the WiFi physical layer. We found that our implementations of $\mathrm{DF}$ and $\mathrm{QMF}$ consistently outperformed AF, as is also the case in theory: the information-theoretical performance of both QMF and DF can achieve the capacity of the one-relay network within 1 $\mathrm{bit} / \mathrm{sec} / \mathrm{Hz}$, which is not the case for AF. Moreover, we found that the performance of QMF and DF is competitive, yet which scheme performs best varies depending on the network configuration, i.e., on the relative strengths of the channels that connect the source, relay and destination. For instance, in a line network, where the source-destination channel is nonexistent, multi-hop DF can outperform QMF; while if in a configuration decoding at the relay fails quite often, QMF can perform much better. Even in a fixed indoor topology, the wireless channels can vary sufficiently to create different configurations over time, and neither QMF nor DF individually can claim to perform universally better across all of them.
To address this challenge, this paper proposes and evaluates QUILT, a system for physical-layer relaying that seamlessly adapts to the underlying network configuration to achieve competitive or better performance as compared to the best current approaches. In QUILT, the relay operates on demand, i.e., is activated only if a first sequence transmitted by the source fails to be decoded by the destination. Once activated, it supports a second transmission of the source through physical layer cooperation. The core component of QUILT is that the relay decides opportunistically whether to use DF or QMF to recover the source sequence, on a frame-by-frame granularity and with no coordination or awareness from the source. Thus QUILT synthesizes on-demand relaying and opportunistic selection of DF or QMF to achieve a consistently good performance across variable configurations.

The relay operates on a very simple principle: it attempts to recover the sequence the source transmitted, interleaves it, and transmits it synchronously with the source. To recover the source sequence, the relay first attempts to decode its received signal, as decoding removes errors and enables to retrieve the clean source sequence (DF operation). Yet, even if the relay cannot decode, the received signal may still contain information useful for the destination: the relay quantizes it to the closest discrete sequence and recovers a noisy (with binary errors) version of the source sequence (QMF operation). In both cases, whether the recovered sequence is error free or not, the relay proceeds to interleave it, to create the sequence it eventually transmits. Interleaving at the relay contributes substantially to the end-to-end QUILT performance, as we show in this work. To understand why, we initiated an extensive theoretical and experimental analysis, and found that interleaving is beneficial mainly for the two reasons that we next discuss.

First, we found that our choice of implementing QMF with interleaving, which was necessitated from practical considerations in [2], can in fact outperform the random mapping proposed in the original QMF scheme [4]. The original information-theoretical version of QMF asks the relay to quantize at the noise level, perform a random mapping and forward the resulting signal; these operations and the analysis assume infinite length and exponentially complex processing. We translated these insights to a practical system by using symbol-level quantization and interleaving of the resulting sequence in [2]. We opted for interleaving as it was the 
only (nontrivial) mapping we found that made decoding with low-complexity belief propagation algorithms possible, while maintaining a simple relay operation. We expected a performance penalty given that interleaving is a very special form of mapping; yet when in this work we compared the performance of interleaving and random mapping, we found to our surprise that interleaving can perform better, at least for one relay network configurations. We performed this comparison using information theoretical analysis and simulation of the two approaches; note that this is the only possible way to compare them, since deploying the original random mapping on a testbed is of prohibitive operational complexity. To this end, in the rest of the paper we use the term QIF to denote the version of QMF with interleaver mapping at the relay.

Second, we found that interleaving is well matched to the OFDM modulation we employ because it enables frequency diversity across subcarriers. Subcarriers separated by more than the coherence bandwidth experience independent channel fading; moreover, interference can be localized in a fraction of the bandwidth the OFDM signal occupies. By interleaving quantized symbols, the relay maps signals received through weak or interfered subcarriers to potentially stronger or cleaner subcarriers in the relay-destination channel, thus realizing frequency diversity. Also note that irrespective of decoding or quantization at the relay, interleaving induces a distributed Space-Frequency Code across the two distributed transmitters (source and relay) and across subcarriers to provide further frequency diversity.

In addition to the novel relay operation, QUILT is also equipped with improved decoding techniques at the destination. The main improvement in the decoder builds on the following observation: even if both the first (unaided) and the second (cooperative) source transmission fail to be decoded by the destination, it is likely that each of them brings some useful information to the decoder and thus, if jointly processed they may lead to successful decoding. In a sense, this approach is the relay-network equivalent of the hybrid-ARQ schemes for point-to-point channels that are fast becoming part of standards [3]. We thus term this "hybrid decoding" and present a low complexity implementation that makes it possible.

In summary, our contributions in this paper are:

- We design QUILT, an on-demand physical layer cooperation scheme of a source with a relay, where the relay retrieves the source sequence by opportunistically decoding or quantizing, interleaves it and transmits it synchronously with the source, while the destination benefits from hybrid decoding.

- We theoretically quantify the effect of our design choices on system performance. Notably, we show that interleaving at the relay outperforms the random mapping that the original QMF information-theoretical scheme dictates.

- We deploy QUILT on a WARPLAB testbed and present exhaustive performance comparisons with DF and QIF protocols through over-the-air experiments. Our experimental results demonstrate benefits up to a factor of 5 for Frame Error Rate (FER) as compared to the next best scheme and two orders of magnitude over the FER of traditional point-to-point transmissions.

The paper is organized as follows: Section II gives an overview of the QUILT components; Section III theoretically justifies our design choices; Section IV describes our system implementation; Section V presents our experimental evaluation; and Section VI discusses related work.

\section{QUILT System OVERVIEW}

QUILT prescribes physical layer operations for a threenode network that consists of a source, a relay, and a destination, building on top of the physical layer procedures of WiFi IEEE802.11. The relay is half-duplex, i.e., it can either transmit or receive. We describe in the following its main components, also depicted schematically in Fig. 1.

\section{A. Source Operation}

Channel Coding: The source takes each information packet and encodes it using an LDPC code (that complies with the IEEE802.11 specifications) to create a coded packet; all transmitted packets by the source are coded. We use coding as recommended in the WiFi standards to increase the end-toend reliability.

Broadband OFDM Modulation: We employ OFDM modulation as specified in the WiFi physical layer to combat channel frequency selectivity. After encoding, the codeword bits are first mapped to QAM symbols and then modulated using OFDM. Each coded packet the source creates results in several OFDM symbols.

\section{B. On Demand Relaying: Two Phase Operation}

Gist: The source first attempts to directly transmit a packet to the destination. The relay also overhears this transmission. If the direct transmission is successful, the source proceeds with the transmission of a new packet; if unsuccessful, the source and the relay cooperatively transmit to try to help the destination decode. We thus have a two-phase operation, where the relay aids the information transfer as-needed, only when the direct transmission from the source is unsuccessful. This enables the system to adapt to the network conditions and avoid unnecessary relay transmissions when the sourcedestination channel is strong.

Signal Exchange: We use vectors $\mathbf{X}=\left[X_{1}, X_{2}, \ldots, X_{m}\right]^{T}$, to collect the QAM symbols transmitted across the $m$ subcarriers of one OFDM symbol. We denote with $\mathbf{X}_{s}[k]$ and $\mathbf{X}_{r}[k]$ the transmitted signal vectors by the source and the relay, $\mathbf{Y}_{r}[k], \mathbf{Y}_{d}[k], \mathbf{Z}_{r}[k]$ and $\mathbf{Z}_{d}[k]$ the received vectors and the Gaussian noise at the relay and destination, and $\mathbf{H}_{i j}[k]=\operatorname{diag}\left(H_{i j, 1}[k], \ldots, H_{i j, m}[k]\right)$ the channel matrix from node $i$ to node $j ; k=1$ or 2 indicates the phase.

- Phase 1: The relay is in listening mode. The received signals per OFDM symbol are:

$$
\begin{aligned}
\mathbf{Y}_{r}[1] & =\mathbf{H}_{s r}[1] \mathbf{X}_{s}[1]+\mathbf{Z}_{r}[1] \\
\mathbf{Y}_{d}[1] & =\mathbf{H}_{s d}[1] \mathbf{X}_{s}[1]+\mathbf{Z}_{d}[1]
\end{aligned}
$$




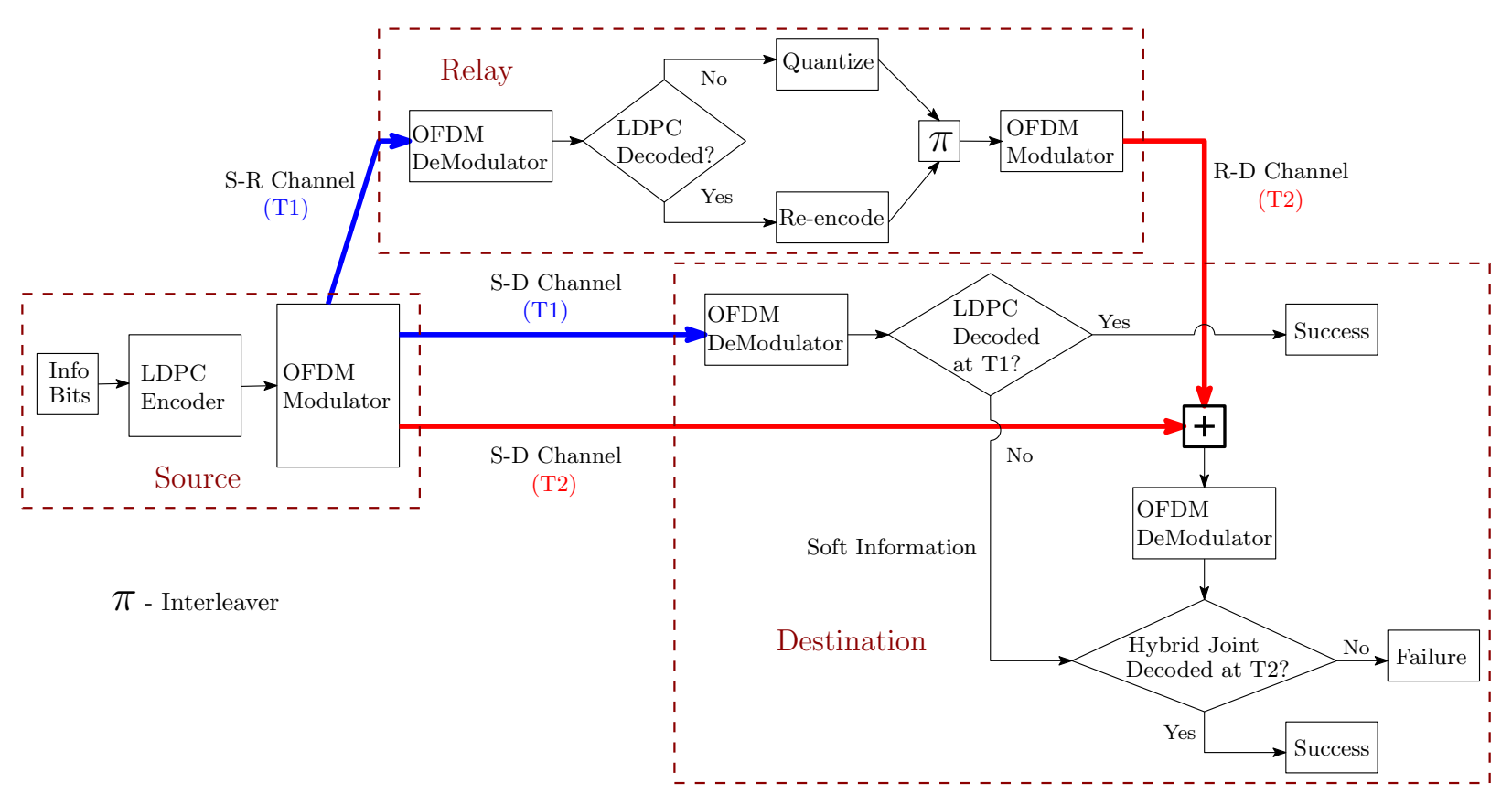

Fig. 1. Schematic diagram of QUILT illustrating the various components of the system. $T_{1}$ and $T_{2}$ indicate the first and second phase, respectively.

If the destination cannot decode we enter Phase 2.

- Phase 2: The source transmits $\mathbf{X}_{s}[2]=\mathbf{X}_{s}[1]$, while the relay transmits a vector $\mathbf{X}_{r}[2]$ that it creates out of $\mathbf{Y}_{r}[1]$ :

$$
\mathbf{Y}_{d}[2]=\mathbf{H}_{s d}[2] \mathbf{X}_{s}[2]+\mathbf{H}_{r d}[2] \mathbf{X}_{r}[2]+\mathbf{Z}_{d}[2] .
$$

How the relay creates $\mathbf{X}_{r}[2]$ is the topic of the next section.

\section{Relay Operation in Phase 2}

At a high level: The relay attempts to decode and exactly recover the source sequence $\mathbf{X}_{s}[1]$; if it fails it uses symbol quantization of the elements of $\mathbf{Y}_{r}[1]$ to their closest constellation points; in both cases, it interleaves the recovered sequence and transmits it synchronously with the source, effectively creating a form of distributed space-frequency coding.

In more detail: The relay operates as follows.

- Attempts to recover the source information, using an LDPC decoder and soft information from its received vectors $\mathbf{Y}_{r}[1]$. It infers success through the CRC check.

- If successful, it re-encodes the source information to create the same vectors $\mathbf{X}_{s}[1]$ as the source.

- If unsuccessful, it quantizes the elements of its received vectors $\mathbf{Y}_{r}[1]$ to their closest constellation points, and creates a (noisy, with discrete errors) version of the $\mathbf{X}_{s}[1]$ vectors the source has.

- Maps the elements of the vectors it has recovered from QAM symbols to bits, interleaves the resulting bit sequence with a randomly selected bit-interleaver, then maps the interleaved bit sequence to signal constellation points, passes it through an OFDM modulator, and transmits it synchronously with the source.
Discussion: We here discuss the reasons for selecting our particular method for sequence recovery, and for interleaving at the relay.

To recover the source sequence, if the relay can successfully decode, this is the optimal operation it can do, as it perfectly cleans up the noise. If the relay fails to decode, our symbol quantization attempts to recover a sequence that is close to the source transmission and conveys information to the destination. To achieve this, symbol quantization is not the only option: infact, the insight from the information theoretic form of QMF is that we should be using sequence quantization. For instance, a possible choice could be to select the codeword an ML decoder would identify, even if this is not the correct one; that is, use the closest codeword to the receiver signal, which amounts to quantizing to the codeword sequences. We were not able to experiment with this option, as it leads to impractical complexity both at the relay and the destination. We opted for symbol quantization that still identifies a sequence close to the transmitted one, yet has viable complexity.

Interleaving is a key component of our relay operation for two independent reasons. The first is specific to OFDM modulation: because of interleaving, the relay assigns signals received through weak or interfered subcarriers in the sourcerelay channel to potentially strong or cleaner subcarriers in the relay-destination channel and also induces mixing of signals from distributed terminals across subcarriers, thus achieving frequency-space diversity and significant performance benefits (see Section V). This benefit is present irrespective of quantization or decoding at the relay. The second reason is specific to QMF: as our theoretical analysis in Section III shows, the mapping that interleaving implements outperforms random mappings for the QMF operation, offering significant 
benefits (see Section III) even when we operate on a single subcarrier; i.e., these benefits are independent of OFDM.

\section{Hybrid Decoding at the Destination}

In phase 1 , the destination attempts to decode using a standard LDPC decoder. If it fails, at the end of phase 2, QUILT takes advantage of the received signals in both phases to decode the source packet. For this, the destination employs a graphical structure that captures the streams received in phases 1 and 2, and adapts to whether decoding or quantization were employed at the relay. The decoder for QUILT is an adaptation of the QIF decoder in [2][5], wherein the stochastic quantizer nodes become deterministic perfect connections if the relay decoding succeeds, and are the same as in [2] otherwise. The decision is guided by a 1-bit flag that the relay transmits, to inform the destination whether the relay-decoding succeeded. Further, the log-likelihood ratio computations take into account the received soft information from both transmission phases.

\section{TheORETICAL ANALYSIS}

We here provide theoretical analysis that substantiates our design choices in QUILT. We show that we gain:

- Benefits from interleaving over the conventional random mapping operation in QMF.

- Benefits from hybrid decoding at the destination.

- Benefits by opportunistic relay decoding or quantization.

For our performance evaluations, we compared information theoretical metrics, such as outage probability, through simulations over narrowband (single-carrier) flat Rayleigh-fading channels that assume infinite complexity processing at the source, the relay and the destination.

\section{A. Performance Metric: Outage Probability}

We evaluate the error performance using the classical notion of outage probability [6], i.e., the probability that a (fixed) transmission rate $R$ is not supported by a scheme. For our calculations we assume 4-QAM constellations at the source and relay. We also assume that the channels are fading i.i.d. over the two phases ${ }^{1}$, independently across the three links, but the distributions in the three links may not be identical. The target rate of the transmit packet is $R=1 \mathrm{bit} / \mathrm{s} / \mathrm{Hz}$. Adapting for our two-phase on-demand relaying protocol, we have that

$$
\begin{aligned}
& \mathbb{P}[\text { Outage }] \\
& =\mathbb{P}\left[\left\{R>\mathrm{C}_{\mathrm{P} 2 \mathrm{P}}\left(h_{s d}[1]\right)\right\} \cap\left\{R>\mathrm{C}_{\mathrm{R}}\left(h_{s d}[1], h_{s r}[1], h_{s d}[2], h_{r d}[2]\right)\right\}\right] \\
& =\mathbb{P}\left[R>\mathrm{C}_{\mathrm{P} 2 \mathrm{P}}\left(h_{s d}[1]\right)\right] \mathbb{P}\left[R>\mathrm{C}_{\mathrm{R}}(\cdot) \mid R>\mathrm{C}_{\mathrm{P} 2 \mathrm{P}}\left(h_{s d}[1]\right)\right]
\end{aligned}
$$

where $\mathrm{C}_{\mathrm{P} 2 \mathrm{P}}\left(h_{s d}[1]\right)$ is the single-user capacity supported by channel $h_{s d}[1]$ and QAM constellation, and $\mathrm{C}_{\mathrm{R}}\left(h_{s d}[1], h_{s r}[1], h_{s d}[2], h_{r d}[2]\right)$ is the capacity of the cooperative scheme, which depends on the particular strategy under consideration. For strategies that do not use hybrid

\footnotetext{
${ }^{1}$ A situation commonly encountered when the two phases occur sufficiently far apart, larger than the coherence time of the channel.
}

decoding, $\mathrm{C}_{\mathrm{R}}$ is just a function of $h_{s r}[1], h_{s d}[2]$ and $h_{r d}[2]$. We evaluate numerically the outage probability by using analytical expressions for $\mathrm{C}_{\mathrm{R}}\left(h_{s r}[1], h_{s d}[2], h_{r d}[2]\right)$ for each strategy, that we derived by modifying the arguments in [4], [7]. The details are outlined in Appendix A.

\section{B. Benefits of Interleaving}

We compare the following schemes: (i) QMF: scalar quantization followed by random mapping at the relay, as in [4], (ii) QIF: scalar quantization followed by bit-level interleaving at the relay and (iii) QF: only scalar quantization at the relay.

The plot in Fig. 2(a) is generated with all three links having i.i.d. Rayleigh fading channels with the same SNR. We observe that QIF outperforms QMF, even for very short interleaver lengths ${ }^{2}$. This can be intuitively explained as follows: in the original QMF relaying scheme, the random mapping at relay results in independence between the transmissions of the source and the relay. Hence the original QMF cannot harness the coherent combining power gain that may increase the performance in the moderate SNR regime. Instead, in QIF the interleaver preserves the weight of the quantized codeword and hence retains certain correlation with the transmission from the source, while providing enough mixing across source and relay terminals to guarantee spatial diversity. Indeed, we observe that QIF outperforms QF significantly, since with no mapping, QF cannot extract the full spatial diversity.

\section{Benefits of Hybrid Decoding}

In Fig. 2(b) (where again all three links have i.i.d. Rayleigh fading channels) we verify that hybrid decoding leads to a significantly improved performance for QIF and QMF. The versions of QMF and QIF with hybrid decoding are labeled QMF-HD and QIF-HD respectively. The gain observed is well expected as the signal received in Phase 1 contains information that can improve the decoding performance. Interestingly, the gain for hybrid decoding in QIF, roughly $1.5 \mathrm{~dB}$, is almost double of that in QMF.

\section{Benefits of Opportunistic Decoding or Quantization}

We compare the following schemes: (i) DF: relay decodes and forwards if it can, else does not cooperate (ii) QIF: as mentioned above, and (iii) DQIF: the relay opportunistically decodes and forwards if possible, else performs QIF.

In Fig. 2(c), where all three links have Rayleigh fading channels, but the SNR in the relay-destination link is four times stronger than that in the source-destination and sourcerelay links, we observe the benefit of opportunistic decoding when the reception at the relay is weak. In particular, while DF slightly outperforms QIF, DQIF is also shown to extract the combined benefits of both DF and QIF. Moreover, we must point out that the theoretical demonstrations for QIF are

\footnotetext{
${ }^{2}$ Due to a multi-letter vector channel representation, it is only feasible to numerically evaluate the expressions for short length interleavers. However, we do see that performance improves with length of the interleaver. Thus the theoretical plots for QIF in this section are much more pessimistic than the long-length interleavers that we use in our over-the-air experiments.
} 


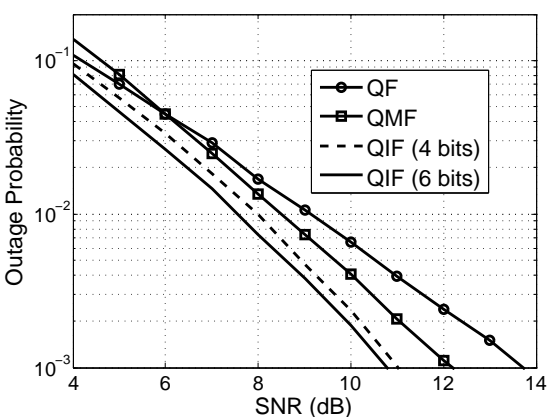

(a) Outage performance for QF, QMF, and QIF. All channels are i.i.d.

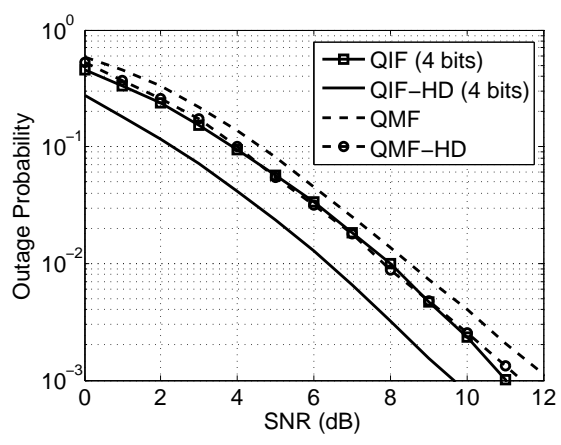

(b) Outage performance for QMF and QIF with a

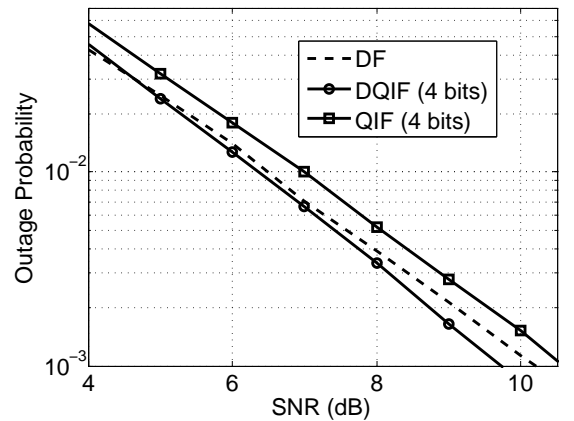

(c) Outage performance for QIF, DF, and DQIF. $\mathrm{SNR}_{R D}=4 \mathrm{SNR}_{S D}=4 \mathrm{SNR}_{S R} . \mathrm{X}$-axis denotes $\mathrm{SNR}_{S D}$

Fig. 2. Outage performance of different relaying schemes.

carried out with short length interleavers and the performance of QIF improves with interleaver length (see Fig. 2(a)). In realworld experiments, we use long interleavers that will provide better performance than the demonstrations in this section show. The relative superiority of DF and QIF will of course vary with channel conditions, but a combination of the two appears to be a promising scheme in terms of universality.

\section{System IMPLEMENTATION}

\section{A. Cooperative Schemes Implemented}

Below we give a description and motivation of the schemes we analyze via experiments using our deployed testbed. The cooperative schemes implemented are summarized in Table I. The relay operations we consider in our experiments are:

- Quantize-Forward $(Q F)$ : Scalar quantization and subsequent forwarding by the relay.

- Quantize-Interleave-Forward (QIF): Scalar quantization followed by bit-level interleaving of the quantized sequences by the relay and subsequent forwarding.

- Decode-Forward (DF): Decoding at the relay if possible and transmit a $2 \times 1$ Alamouti jointly with the source. If decoding at the relay is not possible it remains silent.

- Decode-Interleave-Forward (DIF): Decoding at the relay if possible and transmit bit-level interleaved signal. If decoding at the relay is not possible it remains silent.

- Decode-Interleave-Quantize-Interleave-Forward $(D I Q I F)$ : DIF if relay decoding succeeds; QIF otherwise.

We note that DIF was not considered in our single-carrier theoretical analysis. We implemented this for our (OFDMbased) over-the-air experiments to provide DF an option to exploit the frequency diversity across subcarriers that the interleaver in QIF was inherently providing.

For Phase 2, the destination operations we consider are:

- No Hybrid Decoding: The decoding at destination only uses the signal received in Phase 2.

- On Demand Hybrid Decoding (HD): The destination first attempts to decode with only the signal received in Phase 2. If this decoding fails, then the destination attempts to decode again but this second time with both the signals received in Phase 1 and Phase 2.

To further demonstrate the utility of cooperation, we implement the following baseline scheme:

- Direct Transmission (DT): In this baseline scheme (without the need of a relay) in Phase 2, the source repeats the Phase 1 signal. We also consider DT with the possibility of hybrid decoding, termed DT-HD.

Also, note that, in the nomenclature used in Table I, QUILT refers to DIQIF-HD, which is essentially the all-encompassing system that is the cornerstone of this paper.

\section{B. Frame Structure}

We designed our system to emulate the physical layer procedures of WiFi (IEEE802.11). Each transmitted frame consists of a preamble and the payload. We next describe the preamble and payload fields for the two phases of communication for the schemes we implemented.

1) Preamble: The preamble structure follows what is used in 802.11 systems: it consists of training sequences for Automatic Gain Control (TAGC), training for timing synchronization (TSYNC), and training for channel estimation (TCHE). The training for channel estimation is used to estimate not only the channel but also to estimate the carrier frequency offset.

Phase 1: In this phase, only the source transmits. The preamble structure it transmits is shown in Fig. 3.

Phase 2: In DT, the source transmits the same preamble it transmitted in Phase 1 and the relay remains silent. For all other schemes (QF, QIF, DF, DIF, DIQIF) we deal with joint transmissions from the source and the relay as follows. The TAGC is sent by the source and relay simultaneously. However, we introduce a cyclic shift between the TAGC waveforms sent by the source and the relay to avoid accidental nulling. We send the TSYNC as well as all TCHE fields orthogonally over time, as shown in Fig. 3. Orthogonality for TSYNC ensures that the destination can solve timing synchronization from at least one of two TSYNC sequences, and thus, even if one of the channels happens to be very noisy, it can still synchronize. Orthogonality for TCHE ensures clean 


\begin{tabular}{|c|c|c|c|c|c|}
\hline & $\begin{array}{c}\text { Quantize-Forward } \\
(\mathrm{QF})\end{array}$ & $\begin{array}{c}\text { Quantize-Interleave-Forward } \\
(\mathrm{QIF})\end{array}$ & $\begin{array}{c}\text { Decode-Forward } \\
(\mathrm{DF})\end{array}$ & $\begin{array}{c}\text { Decode-Interleave-Forward } \\
\text { (DIF) }\end{array}$ & $\begin{array}{c}\text { Decode-Interleave- } \\
\text { Quantize-Interleave-Forward } \\
\text { (DIQIF) }\end{array}$ \\
\hline $\begin{array}{c}\text { No } \\
\text { Hybrid } \\
\text { Decoding }\end{array}$ & QF & QIF & DF & DIF & DIQIF \\
\hline $\begin{array}{c}\text { With } \\
\text { Hybrid Decoding } \\
\text { (HD) }\end{array}$ & QF-HD & QIF-HD & DF-HD & DIF-HD & QUILT=DIQIF-HD \\
\hline
\end{tabular}

TABLE I

IMPLEMENTED SCHEMES WHEN THE RELAY IS ACTIVE. THE RELAY OPERATIONS (COLUMNS) AND THE DESTINATION OPERATIONS IN PHASE 2 (ROWS), ARE DESCRIBED IN SECTION IV-A.

channel estimates for separate links (a similar approach is used for MIMO channel training implementations).

2) Payload: The payload consists of OFDM symbols, i.e., contains data and pilot subcarriers as described in 802.11.

Phase 1: For all the schemes, we transmit the exact same payload waveform, which corresponds to an OFDM-based single transmitter single receiver antenna system.

Phase 2: In DT, the source transmits the same payload it transmitted in Phase 1 and the relay remains silent. In QF, QIF, DIF and DIQIF, the source retransmits the same payload it transmitted in Phase 1, and the relay transmits its received and processed signal. In the DF, if the relay has successfully decoded (the CRC passed), we have the source and relay payload implement a $2 \times 1$-antenna distributed Alamouti code to provide spatial diversity as in [2]. In DF and DIF, if the relay cannot decode then it remains silent.

In QF, QIF and DIQIF schemes, the payload contains one more OFDM symbol than the DF and DIF schemes which is only sent by the relay. This extra OFDM symbol is used to forward an estimate of the source-relay SNR to the destination, which needs to employ it during iterative decoding. The relay first estimates the SNR and quantizes it to one of 40 possible values ranging from -10 to $30 \mathrm{~dB}$ (in steps of $1 \mathrm{~dB}$ ). We can describe these 40 values using 6 bits. For QF and QIF we repeat these 6 bits 8 times, modulate them with BPSK and allocate them to 48 data subcarriers in the OFDM symbol used to forward the SNR information. For DIQIF, in addition to the 6 bits of SNR information, we also send one extra bit to the destination to notify if the relay decoded successfully or not. The 7 bits for DIQIF are sent in the 48 data subcarriers of the extra OFDM symbol.

We note that, for decoding the payload, an estimation of the effective noise variance is required by the LDPC decoder for computation of the log-likelihood ratios. For the estimation of SNR and effective noise variance, we follow the same approach as presented in [2].

As per the 802.11 standard, each OFDM symbol in the payload consists of a total of 48 data subcarriers, 4 pilot subcarriers and 12 unused subcarriers. The 4 pilot subcarriers are used for residual phase noise and CFO correction. For the joint source and relay transmissions, we synchronize the carrier and timing between the source and relay by sharing a wire

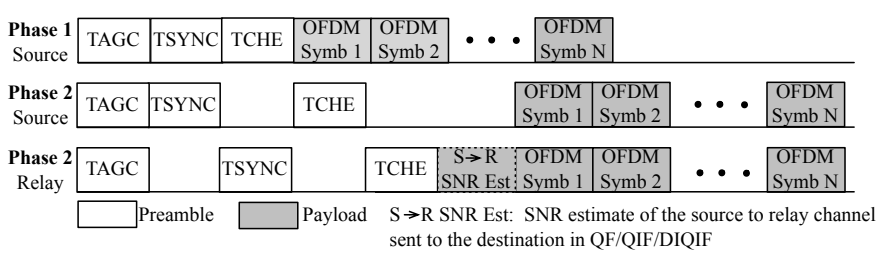

Fig. 3. Time diagram.

connection between them as shown in Fig. 4. Yet, we would like to mention that recent work on distributed transmissions has shown that it possible to also achieve accurate timing and carrier synchronization in a distributed manner (see [8], [9], [10]); these protocols are enabled by implementing a large part of the mechanisms in real time in the FPGA to achieve fast turnaround times. Incorporating this into WARPLab, although feasible, was not our focus.

\section{EXPERIMENTAL EVALUATION}

In this section, we experimentally evaluate QUILT and compare it with alternative cooperative communication strategies. We first describe our performance metrics (Section V-A) and testbed (Section V-B), then present our experimental results (Sections V-C, V-D, V-E and V-F).

\section{A. Performance Metrics}

We consider the following metrics:

- Frame-Error Rate (FER), represents the percentage of source packets that were not decoded after both phases.

- Throughput, measures the number of information bits successfully delivered to the destination per channel use (bps/Hz).

\section{B. Testbed}

We used the WARP SDR hardware to implement the source, relay and destination nodes in our testbed. We used the WARPLab framework to interact with the WARP hardware via a host PC running MATLAB. The host PC was connected to the nodes via an Ethernet switch as shown in Figure 4. The samples to be transmitted by a node were generated in MATLAB and downloaded to the transmit buffer of the 


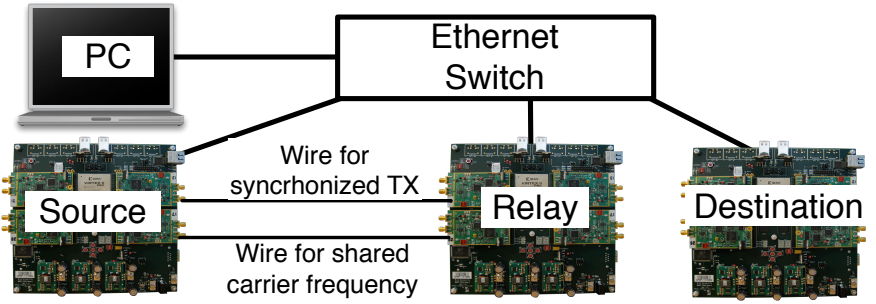

Fig. 4. Node and host PC configuration

corresponding node. The host PC triggered a real-time overthe-air transmission and reception by the nodes. The samples received at a node were read by the host PC and processed in MATLAB. The transmitted waveforms were centered at $2.4 \mathrm{GHz}$ and had a $20 \mathrm{MHz}$ bandwidth.

We evaluate the performance of the protocols for different experiment scenarios which were obtained by keeping the source fixed and varying the relay and destination placement and source and relay powers. The node locations for each of the three scenarios considered are shown in Fig. 5 and the Received Signal Strength Indicator (RSSI) for each link for each scenario is shown in Fig. 6.

For each setting, we ran the experiment for at least 2500 coded frames. In all experiments, we used randomly chosen bit-interleavers of length equal to that of an LDPC codeword. We used 16-QAM constellations with a coding rate of $3 / 4$.

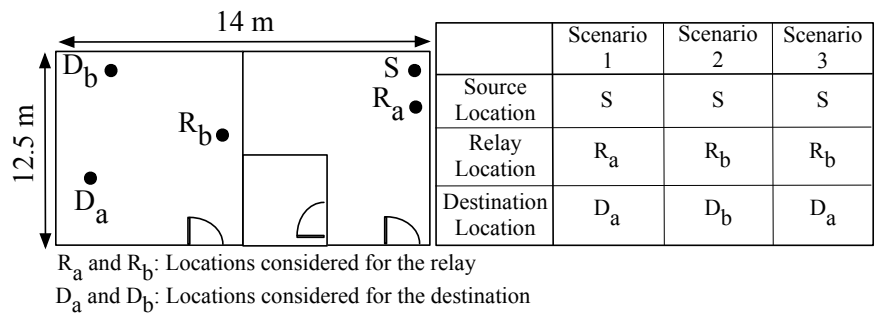

Fig. 5. Node placement illustrating the topologies considered.

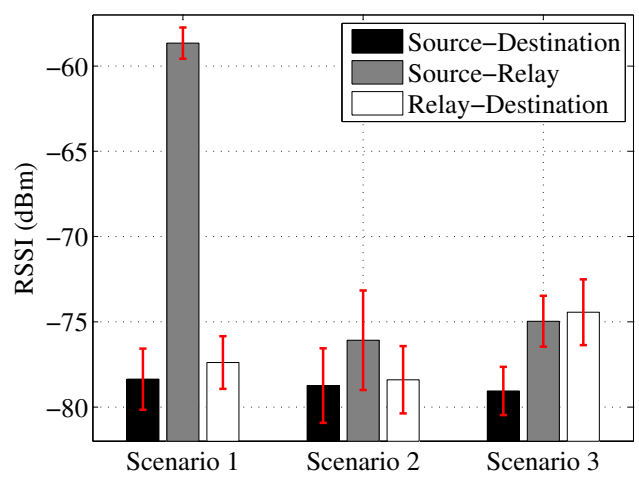

Fig. 6. RSSIs for the different settings considered.

\section{Evaluation of Interleaving}

We observed in Section III that interleaving can significantly improve the outage probability of QIF vs. $\mathrm{QF}^{3}$ (see Figure 2(a)). The theoretical evaluation was only possible for short interleavers, and across a single subcarrier. The question is: how much interleaving helps when we use long interleavers across subcarriers?

Fig. 7(a) and 8(a) present the performance of DF, QF, DIF and QIF. We note that for these experiments, we allowed DF to implement an Alamouti code when the source and the relay cooperatively transmit in the phase 2 , thus achieving full spatial diversity. We make the following observations.

First, QIF outperforms QF in all three scenarios, with throughput gains ranging from $\mathbf{1 5} \%$ to $\mathbf{3 0} \%$. We expected significant benefits, as interleaving enables to capture spacefrequency diversity. Infact, it was shown in [5] that interleaving is sufficient to extract full spatial diversity from distributed transmissions for single carrier systems; here, we have the additional benefit of capturing frequency diversity through mixing signals across OFDM subcarriers.

Second, although DF achieves full spatial diversity thanks to the Alamouti code, DIF can still offer benefits, up to an impressive $\mathbf{4 5} \%$ throughput gain (in Scenario 2, Fig. 8(a)). Since DF already achieves full spatial diversity, these benefits reflect the frequency diversity that interleaving offers in addition to the spatial diversity.

Third, Scenario 1 provides evidence that DIF can in some cases outperform QIF.

\section{Evaluation of Hybrid Decoding}

Next, we investigate the effect of relay-assisted hybrid decoding. Fig. 7(b) and 8(b) compare the performance of DIF and QIF, which in the second phase utilize only the second transmission for decoding, with that of DIF-HD and QIF-HD, which combine the received signals in both phases 1 and 2 when decoding. We observe that:

First, hybrid decoding consistently offers benefits for both QIF and DIF across all the three scenarios, for instance up to 25 times FER improvement (in Scenario 1, Fig. 7(b)).

Second, hybrid decoding makes a more significant difference when the channels are less noisy, i.e., we start with lower FER, as is the case in Scenario 1. This is because there are comparatively fewer errors in the erroneous codewords, which can be corrected with hybrid decoding.

Third, hybrid decoding can help QIF more than DIF, as we see in Scenarios 2 and 3. This is because with DIF, when the relay cannot decode it remains silent in phase 2; while with QIF the relay always transmits potentially useful information that can be leveraged through hybrid decoding across both phases, which is reflected in the QIF-HD performance.

\footnotetext{
${ }^{3} \mathrm{We}$ emphasize once again that the random mapping version of QMF in [4] is not an implementable strategy due to complexity limitations. Moreover, we have shown in Section III that QIF outperforms random mapping.
} 


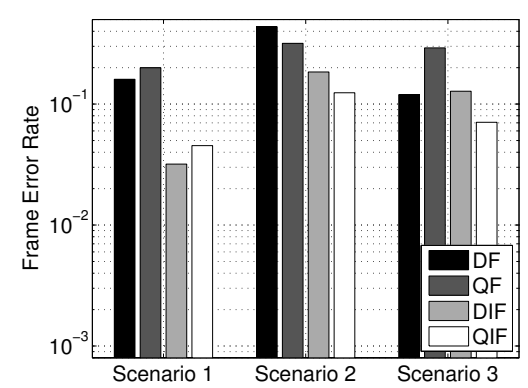

(a) FER benefits of interleaving.

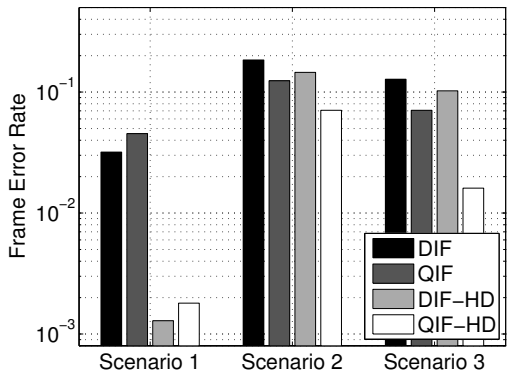

(b) FER benefits of hybrid decoding.

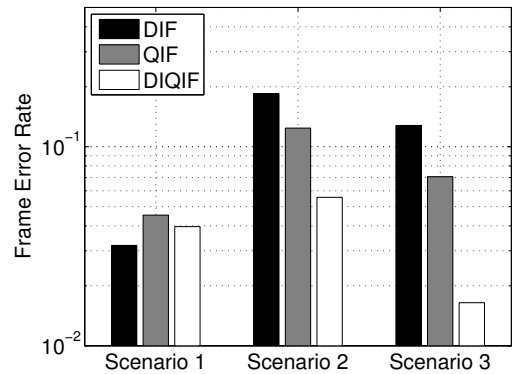

(c) FER benefits of opportunistic decoding.

Fig. 7. FER benefits of interleaving, hybrid decoding, and opportunistic decoding.

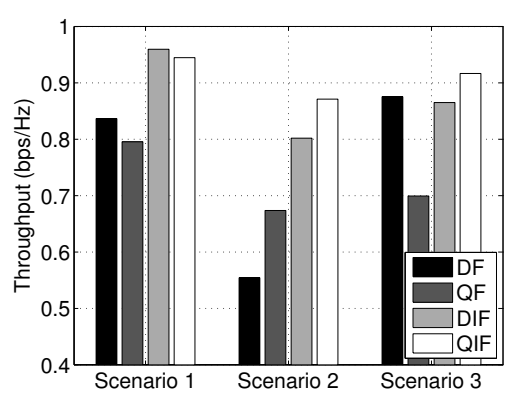

(a) Throughput benefits of interleaving.

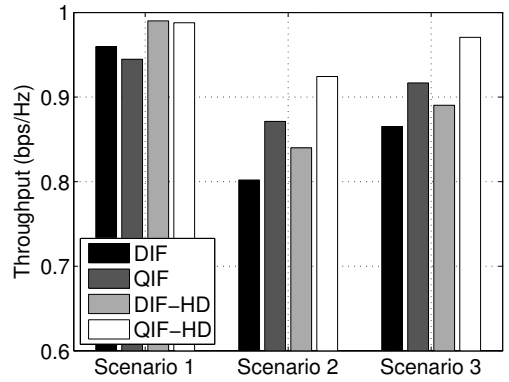

(b) Throughput benefits of hybrid decoding.

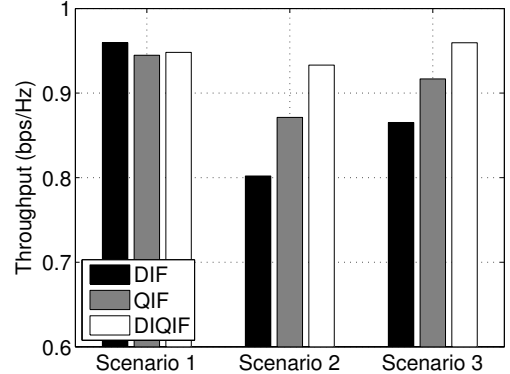

(c) Throughput benefits of opportunistic decoding.

Fig. 8. Throughput benefits of interleaving, hybrid decoding, and opportunistic decoding/quantizing.

\section{E. Evaluation of Opportunistic Decoding or Quantizing}

To explore the performance of opportunistically decoding or quantizing at the relay, Fig. 7(c) and 8(c) compare the FER and throughput performance of DIF and QIF vs. DIQIF. We find that:

DIQIF, that implements opportunistic decoding/quantizing, has competitive or better performance than the next best scheme, as high as a factor of $\mathbf{8}$ over DIF and a factor of $\mathbf{5}$ over QIF (as in Scenario 3, Fig. 7(c)). The benefits of DIQIF are more pronounced when the source-to-relay link is weak, as is the case in Scenarios 2 and 3. This is because, in such cases the relay cannot decode, and DIF cannot exploit the relaydestination channel, while DIQIF can. Moreover, although QIF outperforms DIF in terms of FER, there exist frames where relay decoding is possible, and the opportunistic DIQIF decoding enables to clean them up from the source-relay noise, thus boosting the end-to-end performance. In Scenario 1, on the other hand, the source-relay link is very strong and supports relay decoding almost all the time; the DIQIF relay also performs decoding, but has the added requirement of communicating a 1-bit flag to inform the destination whether it decoded; we believe it is errors in this bit that result in the marginal penalty of the DIQIF performance over DIF.

\section{F. Putting it All Together: Evaluation of QUILT}

We compare in Fig. 9(a) the FER performance of QUILT with (i) DIF-HD and QIF-HD, the most competing strategies implemented in this paper, and (ii) DT-HD, direct transmissions with hybrid decoding, to benchmark the performance of a system without a relay. We observe the following:

First, we note FER gains of over 2 orders of magnitude of our relaying strategies vs. DT-HD (in Scenario 1, Fig. 9(a)), clearly illustrating the benefits of relaying.

Second, QUILT has competitive or better performance than the next best scheme, up to a factor of 5 over QIF-HD (in Scenario 3, Fig. 9(a)). In Scenario 1, where the source-relay link is very strong, we observed very few errors for DIF-HD, QIF-HD and QUILT (even after running the experiments in this scenario for over 4000 frames) as hybrid decoding cleans up most errors in this setup, leading to similar performance across the three schemes (marginally better for QUILT).

Since we operate at quite low FERs, we note that the vast majority of transmissions are successful and thus, the difference in fraction of frames correctly decoded does not lead to discernible throughput differences in Fig. 9(b). However, when operating at higher FERs, we believe that the FERs trends evidenced in Fig. 9(a) will lead to more significant throughput differences, as was the case in Fig. 8(b), 8(c).

Overall, we find that QUILT, by synthesizing opportunistic selection of decoding/quantizing and interleaving at the relay with hybrid decoding at the destination, achieves universally competitive performance across all the scenarios we examined. 


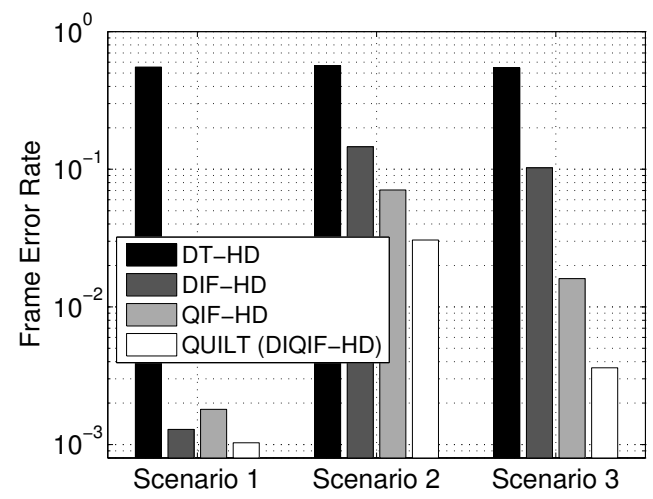

(a) FER benefits of QUILT.

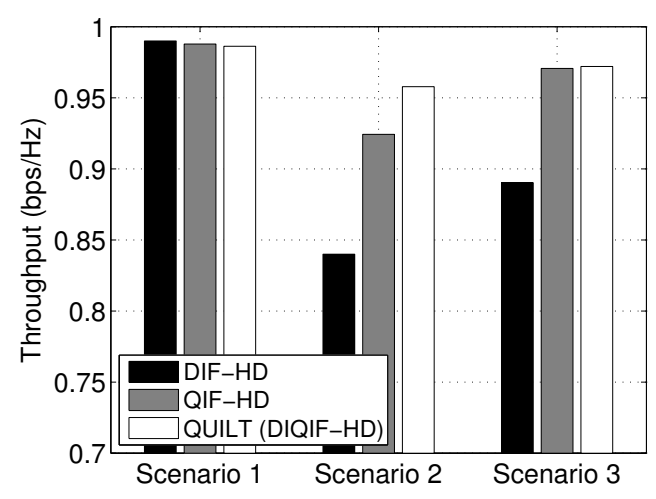

(b) Throughput benefits of QUILT.

Fig. 9. Performance of QUILT.

\section{RELATED WORK}

Our paper in [2] presented a first implementation of QIF, and offered comparisons with DF and AF relaying schemes. Although our work builds on [2], QUILT differs in a number of important features, that include: (a) the opportunistic use of decoding or quantizing at the relay; (b) the use of interleaving even when decoding was successful; and (c) the use of hybrid decoding. Completely new to this work is also the theoretical analysis that illustrates through outage calculations the benefits of interleaving and hybrid decoding, as well as all the experimental evaluations and comparisons. In summary, all claimed novel contributions are unique to this paper.

The works in [11], [12] survey testbed implementations of physical layer relay schemes; the focus is on the implementation of either DF or AF schemes. A testbed based on uncoded DF in a single-relay system was investigated in [13]. A WARP radio testbed based on DF was implemented in [14]. None of these works implemented advanced error correction or broadband OFDM modulation. In [8] both (uncoded) $\mathrm{AF}$ and $\mathrm{DF}$ relaying along with distributed Alamoutibased transmission were implemented over broadband OFDM. However, this implementation lacked error correcting codes and distributed frequency-diversity coding. Apart from the relaying strategy, other issues related cooperative relaying have also been studied through implementation on testbeds; for example the experimental work in [9] and [15] focuses on the synchronization for multiple simultaneous transmissions.

The monograph in [1] surveys the recent theoretical development in cooperative relaying. QMF was originally proposed in [4] for Gaussian networks and shown to approximately achieve the network capacity. It was later extended to discrete memoryless networks in [7]. Practical coding schemes of QMF relaying with LDPC and BICM were proposed for a halfduplex single-relay cooperative MIMO system in [16] and for a full-duplex multi-relay network in [5], where in the latter interleavers as relay mapping were first proposed. The use of demodulation instead of quantization was used in [5] and [17]. None of these papers had an experimental evaluation.

\section{REFERENCES}

[1] G. Kramer, I. Marić, and R. Yates, "Cooperative communications," Found and Trends in Networking, vol. 1, no. 3, pp. 271-425, 2006.

[2] M. Duarte, A. Sengupta, S. Brahma, C. Fragouli, and S. Diggavi, "Quantize-map-forward (QMF) relaying: An experimental study," in Proceedings of the ACM MobiHoc, July 2013.

[3] "Local and metropolitan area networks-specific requirements part 11: Wireless LAN medium access control (MAC) and physical layer (PHY) specifications," IEEE Std 802.11-2012.

[4] A. S. Avestimehr, S. N. Diggavi, and D. N. C. Tse, "Wireless network information flow: A deterministic approach," IEEE Transactions on Information Theory, vol. 57, no. 4, pp. 1872-1905, April 2011.

[5] A. Sengupta, S. Brahma, A. Ozgur, C. Fragouli, and S. Diggavi, "Graphbased codes for quantize-map-and-forward relaying," in Proceedings of the IEEE Information Theory Workshop, October 2011, pp. 140-144.

[6] D. Tse and P. Viswanath, Fundamentals of Wireless Communication. Cambridge University Press, May 2005.

[7] S. H. Lim, Y.-H. Kim, A. E. Gamal, and S.-Y. Chung, "Noisy network coding," IEEE Transactions on Information Theory, vol. 57, no. 5, pp. 3132-3152, May 2011.

[8] P. Murphy and A. Sabharwal, "Design, implementation, and characterization of a cooperative communications system," IEEE Transactions on Vehicular Technology, vol. 60, no. 6, pp. 2534-2544, July 2011.

[9] H. Rahul, H. Hassanieh, and D. Katabi, "SourceSync: a distributed wireless architecture for exploiting sender diversity," in Proceedings of the ACM SIGCOMM, August 2010, pp. 171-182.

[10] H. V. Balan, R. Rogalin, A. Michaloliakos, K. Psounis, and G. Caire, "AirSync: Enabling distributed multiuser MIMO with full spatial multiplexing," IEEE/ACM Transactions on Networking, no. 99, 2013.

[11] T. Korakis, M. Knox, E. Erkip, and S. Panwar, "Cooperative network implementation using open-source platforms," IEEE Communications Magazine, vol. 47, no. 2, pp. 134-141, 2009.

[12] G. Bradford and J. N. Laneman, "A survey of implementation efforts and experimental design for cooperative communications," in Proceedings of the IEEE ICASSP, March 2010, pp. 5602-5605.

[13] _ "An experimental framework for the evaluation of cooperative diversity," in Proceedings of the IEEE CISS, March 2009, pp. 641-645.

[14] M. Knox and E. Erkip, "Implementation of cooperative communications using software defined radios," in Proceedings of the IEEE ICASSP, March 2010, pp. 5618-5621.

[15] X. Zhang and K. G. Shin, "DAC: Distributed asynchronous cooperation for wireless relay networks," in Proceedings of the IEEE INFOCOM, March 2010, pp. 1064-1072.

[16] V. Nagpal, I.-H. Wang, M. Jorgovanovic, D. Tse, and B. Nikolić, "Coding and system design for quantize-map-and-forward relaying," IEEE Journal on Selected Areas in Communications, August 2013, see also arXiv: 1209.4679 [cs.IT].

[17] E. Atsan, R. Knopp, S. Diggavi, and C. Fragouli, "Towards integrating quantize-map-forward relaying into LTE," in Proceedings of the IEEE Information Theory Workshop, September 2012, pp. 212-216. 


\section{APPENDIX A}

\section{Outage Probabilities of CoOperative Schemes}

Quantized Forwarding $(Q F)$ : In the absence of relayassisted hybrid decoding, we have,

$$
\mathrm{C}_{\mathrm{R}}^{\mathrm{QF}}=I(X ; Y[2]),
$$

where $I(\cdot ; \cdot)$ denotes the mutual information. Since the relay quantizes its received signal, the overall transformation of the source signal can be represented as an end-to-end channel whose capacity can be evaluated as above. This capacity computation can be done numerically as no closed form expressions exist for such (scalar) quantized channels with QAM inputs. For hybrid decoding, the achievable rate can be evaluated as,

$$
\mathrm{C}_{\mathrm{R}}^{\mathrm{QF}}=I(X ; Y[1], Y[2]),
$$

which can then be again numerically computed to yield the outage probability by using (3).

Quantize-Interleave-Forward $(Q I F)$ : We evaluate an interleaver that operates over a block of length $K$ as follows:

$$
\mathbf{X}_{\mathrm{R}}=\Pi\left(\hat{\mathbf{Y}}_{\mathrm{R}}\right)
$$

where $\Pi$ denotes a specific permutation on the quantized sequence $\hat{Y}_{\mathrm{R}}$. This permuted sequence is transmitted by the relay. We can numerically evaluate the rate for the interleaved scheme by using $K$-letter mutual information characterization, which is similar to a vector version of (4)-(5) while including the aforementioned interleaver operation.

Quantize-Map-Forward $(Q M F)::$ In the originally proposed information-theoretic QMF [4], the mapping codebook at the relay is generated randomly . The analytical result in [4] can be used to evaluate outage probability after doing a simple generalization to QAM constellations for transmission and quantization. We can then numerically evaluate the achievable rate. This can be done for both the link cooperation scheme as well as hybrid decoding.

Decode-Forward (DF): In Phase 2, if the relay can decode from its Phase 1 reception, it re-encodes the decoded message and transmit it, so that coherent cooperation is attained. If the relay cannot decode, it keeps silent. The outage event can be evaluated as follows.

$$
\begin{aligned}
& \text { Outage } \Longleftrightarrow \\
& \left\{R>\mathrm{C}_{\mathrm{P} 2 \mathrm{P}}(h[1])\right\} \cap\left\{\left\{R \leq \mathrm{C}_{\mathrm{P} 2 \mathrm{P}}\left(h_{r}[1]\right) \text { and } R>\mathrm{C}_{\mathrm{MISO}}(h[2], g[2])\right\} \cup\right. \\
& \left.\left\{R>\mathrm{C}_{\mathrm{P} 2 \mathrm{P}}\left(h_{r}[1]\right) \text { and } R>\mathrm{C}_{\mathrm{P} 2 \mathrm{P}}(h[2])\right\}\right\}
\end{aligned}
$$

Opportunistic-Decoding QIF (DQIF): A natural way to combine QIF and DF relaying is the following: if the relay can decode, it performs DF as above; otherwise, it performs QIF instead of keeping silent.
With this opportunistic scheme at the relay, the outage event can be evaluated as follows.

$$
\begin{aligned}
& \text { Outage } \Longleftrightarrow \\
& \left\{R>\mathrm{C}_{\mathrm{P} 2 \mathrm{P}}(h[1])\right\} \cap\left\{\left\{R \leq \mathrm{C}_{\mathrm{P} 2 \mathrm{P}}\left(h_{r}[1]\right) \text { and } R>\mathrm{C}_{\mathrm{MISO}}(h[2], g[2])\right\} \cup\right. \\
& \left.\left\{R>\mathrm{C}_{\mathrm{P} 2 \mathrm{P}}\left(h_{r}[1]\right) \text { and } R>\mathrm{C}_{\mathrm{R}}^{\mathrm{QIF}}\left(h_{r}[1], h[2], g[2]\right)\right\}\right\}
\end{aligned}
$$

